\title{
Interval Reliability Analysis of Titanium Alloy Fatigue
}

\author{
Benyang Tu, Chunyang Zhao
}

\begin{abstract}
The fatigue crack growth process of titanium alloy is affected by uncertain factors, which leads to uncertainty in the parameters of fatigue analysis, and it is necessary to carry out reliability analysis. Aiming at the limitations of the probabilistic reliability method on the high sample size and statistical information and the large computational complexity, a non-probabilistic reliability analysis method for fatigue crack growth interval of titanium alloy is proposed. The uncertain parameters in the Paris model are expressed as intervals, the interval non-probabilistic reliability index of fatigue crack propagation is defined and considered based on the fatigue life model characterizes, and uses the interval mathematics theory to solve the non-probabilistic reliability index. The example calculation results show that the non-probability reliability index decreases linearly from $99.231 \%$ to $90.846 \%$, and the calculation result is more conservative than the probability reliability method, which is safer in engineering applications.
\end{abstract}

Index Terms - titanium alloys; crack growth; interval variables ; non-probabilistic reliability; fatigue life

\section{INTRODUCTION}

Titanium alloy materials are widely used in pressure-resistant shell structures. The pressure-resistant shell structure will withstand the cyclical reciprocating stress of seawater during service[1], and the resulting fatigue damage may cause cracks and become an important factor affecting structural safety. Therefore, the analysis of fatigue fracture of titanium alloy has always been a research hotspot in engineering. The existence of cracks greatly reduces the strength of the structure, resulting in fracture stress far less than the yield stress, even less than the allowable stress[2], is the root cause of fracture failure. In fatigue problems[3], fatigue load, fatigue life, fatigue crack growth rate and other data often have great randomness and dispersion. In view of the uncertainty in fatigue problems, it is necessary to analyze the crack propagation using reliability theory. The traditional structural fatigue reliability analysis using probabilistic fracture mechanics [4] requires accurate description of the probability distribution of uncertain parameters in the reliability model, while the actual engineering and experimental data volume is often lacking[5], which is not easy to obtain. Uncertain statistical data of uncertain parameters, small errors of the probability model can lead to large errors in the reliability calculation results[6], so the

Benyang Tu Postgraduate of Naval Architecture and Ocean En-gineering department of Jiangsu University of Science and Technology,China.+8618260636263

Chunyang Zhao Postgraduate of Naval Architecture and Ocean En-gineering department of Jiangsu University of Science and Technology, China.+8618796087050 accuracy of the reliability analysis results cannot be guaranteed.

In order to solve the reliability analysis problem in the absence of statistical data, some scholars have proposed a non-probabilistic reliability analysis method, and carried out some research on engineering applications. Ben-Haim[6] and Elishakoff[7] first proposed an interval-based non-probabilistic reliability analysis method, using interval numbers to describe unknown but bounded uncertain parameters, and using interval operations for analysis, the method only needs to determine uncertainty. The upper and lower bounds of the parameter. Guo Shuxiang et al.[8] used interval variables to describe structural uncertain parameters, and proposed a non-probabilistic reliability index and analysis method based on interval analysis, considering whether the structural stress set and the structural strength set interfere. Wang Xiaojun[9] established a structural stress-strength non-probability set interference model, which uses the ratio of the volume of the structural safety domain to the total volume of the basic interval variable domain as a measure of the structural non-probabilistic reliability. Qiu Zhiping[10] and Jiang Chao[11] gave a detailed summary and summary of the interval-based algorithm, which provided a reference for the calculation in engineering. Sun Wencai[2] discussed the non-probabilistic reliability metrics. The interval non-probabilistic reliability analysis method was used to analyze the fracture mechanics based on fracture mechanics. The Monte-Carlo simulation algorithm was used to verify the reliability. Fu Anxi[13] uses the interval variable to describe the design parameters of the manned spherical shell structure. The structural reliability index is characterized by the satisfaction degree of the ultimate load interval greater than or equal to the load range. The three algorithms are used to describe the manned spherical shell. Non-probabilistic reliability analysis also provides ideas for this article.

In this paper, the interval analysis theory is applied to the fatigue crack propagation reliability analysis of titanium alloy. The uncertainty parameter in the crack propagation model is used as the interval variable. Combined with the crack propagation calculation formula and the fatigue life reliability model, the non-probability of fatigue crack propagation is established. Limit state equation for reliability. Based on the interval non-probabilistic reliability theory, the interval probability of fatigue crack growth life greater than or equal to the fatigue crack propagation design life is taken as the non-probabilistic reliability index. The interval arithmetic method is used to calculate the reliability index, and compared with the probability reliability method. The non-probability method is more conservative, and it is safer in engineering applications. The feasibility of this method is verified. The method is simple and intuitive, and the fatigue reliability analysis is easy and quick. 


\section{INTERVAL MATHEMATICS}

\section{A. Interval number and its algorithm}

The interval number is a representation of the endpoint of the bounded real number set[11]. Suppose that the upper and lower bounds of the real set are respectively $\bar{x}$ and $\underline{x}$, and the closed bound real set can be expressed as:

$$
X^{I}=[\underline{x}, \bar{x}]=\{x \mid x \in R, \underline{x} \leq x \leq \bar{x}\}
$$

The above formula is called the bounded closed interval, where $\bar{x}$ is the endpoint on the interval and $\underline{x}$ is the endpoint at the interval.In the bounded closed interval, it is called the interval variable; $X^{I}$ is called the interval number, which is the interval where the variable $x$ is located; let the set of all the intervals on the real number $\mathrm{R}$ be $I(R)$.

The number of spaces has some common basic quantities that reflect its geometric properties. The width $\omega\left(X^{I}\right)$, the dispersion $x^{r}$, the mean $x^{c}$, and the relative uncertainty $\beta\left(X^{I}\right)$ of the interval number $X^{I}$ are expressed as:

$$
\begin{aligned}
& \omega\left(X^{I}\right)=\bar{x}-\underline{x} \\
& x^{r}=\frac{\bar{x}-\underline{x}}{2}=\frac{1}{2} \omega\left(X^{I}\right) \\
& x^{c}=\frac{\underline{x}+\bar{x}}{2} \\
& \beta\left(X^{I}\right)=\frac{x^{r}}{\left|x^{c}\right|}=\frac{\bar{x}-\underline{x}}{|\bar{x}+\underline{x}|}
\end{aligned}
$$

The calculation of the interval number is based on the algorithm of the set. For the interval numbers $X^{I}=[\underline{x}, \bar{x}]$ and $Y^{I}=[y, \bar{y}]$, the addition and subtraction of the two interval numbers are added and subtracted between all the elements in the two interval numbers, and the upper and lower bounds of the interval numbers obtained by multiplying the interval numbers are respectively asked. The maximum and minimum values of the endpoints of the two interval numbers, and the number of intervals can be regarded as the inverse of the multiplication[12]. In summary, the interval four algorithm is organized as follows:

$$
\begin{gathered}
X^{I}+Y^{I}=[\underline{x}, \bar{x}]+[\underline{y}, \bar{y}]=[\underline{x}+\underline{y}, \bar{x}+\bar{y}] \\
X^{I}-Y^{I}=[\underline{x}, \bar{x}]-[\underline{y}, \bar{y}]=[\underline{x}-\bar{y}, \bar{x}-\underline{y}] \\
X^{I} \times Y^{I}=[\underline{x}, \bar{x}] \times[\underline{y}, \bar{y}]=([\min (S), \max (S)], \\
S=\underline{x} \underline{y}, \underline{x} \bar{y}, \bar{x} \underline{y}, \bar{x} \bar{y}) \\
X^{I / Y^{I}}=[\underline{x}, \bar{x}] \div[\underline{y}, \bar{y}] \\
=[\underline{x}, \bar{x}] \times\left[\frac{1}{\bar{y}}, \frac{1}{\underline{y}}\right](0 \notin[\underline{y}, \bar{y}])
\end{gathered}
$$

\section{B. The possibility of interval inequality}

The size between the two real numbers can be directly judged by the numerical value, and the absolute value between the two interval numbers cannot be directly judged by a single value. The inequality relationship between the interval numbers is generally measured by the interval possibility[13]. Ishibuchi and Tanaka[14] proposed the following definitions of the two possible intervals:

1) Interval probability that interval number $X^{I}=[\underline{x}, \bar{x}]$ is greater than or equal to real number (point interval) $\alpha$

$$
P\left(X^{I} \leq \alpha\right)=\max \left\{0, \min \left\{1, \frac{\alpha-\underline{x}}{\omega\left(X^{I}\right)}\right\}\right\}
$$

2) The probability that the interval number $Y^{I}=[\underline{y}, \bar{y}]$ is greater than or equal to the interval number of $X^{I}=[\underline{x}, \bar{x}]$

$$
P\left(Y^{I} \geq X^{I}\right)=\max \left\{0, \min \left\{1, \frac{\bar{y}-\underline{x}}{\omega\left(X^{I}\right)+\omega\left(Y^{I}\right)}\right\}\right\}
$$

Jiang Chao[11] summarized the six possible relationships between the two intervals, combined with the probability idea, and proposed a unified interval probability. The interval of this paper is based on this method for interval probability calculation, which is expressed as:

$$
P\left(Y^{I} \geq X^{I}\right)=\left\{\begin{array}{cc}
0, & \underline{x} \geq \bar{y} \\
0.5 \cdot \frac{\bar{y}-\underline{x}}{\omega\left(X^{I}\right)} \cdot \frac{\bar{y}-\underline{x}}{\omega\left(Y^{I}\right)}, & \underline{y} \leq \underline{x} \leq \bar{y} \leq \bar{x} \\
\frac{\underline{y}-\underline{x}}{\omega\left(X^{I}\right)}+0.5 \frac{\omega\left(Y^{I}\right)}{\omega\left(Y^{I}\right)} & \underline{x} \leq \underline{y} \leq \bar{y} \leq \bar{x} \\
\frac{y-\underline{x}}{\omega\left(X^{I}\right)}+\frac{\bar{x}-\underline{y}}{\omega\left(X^{I}\right)} \cdot \frac{\bar{y}-\bar{x}}{\omega\left(Y^{I}\right)}+0.5 \cdot \frac{\bar{x}-\underline{y}}{\omega\left(X^{I}\right)} \cdot \frac{\bar{x}-\underline{y}}{\omega\left(Y^{I}\right)}, \underline{x}<\underline{y} \leq \bar{x}<\bar{y} \\
\frac{\bar{y}-\bar{x}}{\omega\left(Y^{I}\right)}+0.5 \frac{\omega\left(X^{I}\right)}{\omega\left(Y^{I}\right)}, & \underline{y} \leq \underline{x}<\bar{x}<\bar{y} \\
1, & \bar{x}<\underline{y}
\end{array}\right.
$$

\section{NON-PROBABILISTIC RELIABILITY ANALYSIS OF CRACK} PROPAGATION IN TITANIUM ALLOY BASED ON INTERVAL

\section{A. Crack propagation model and establishment of limit state equation}

At present, in the study of fatigue crack propagation of titanium alloy based on fracture mechanics, the most widely used Paris crack propagation formula is expressed as:

$$
\frac{d a}{d N}=C(\Delta K)^{n}
$$

Where $a$ is the crack length, $N$ is the number of crack propagation cycles, $d a / d N$ is the crack growth rate, $C$ and n are material constants, and $\Delta K$ is the range of stress intensity factors in the vicinity of the crack. The expression is:

$$
\Delta K=F \Delta \sigma \sqrt{\pi a}=F \sqrt{\pi a}\left(\sigma_{\max }-\sigma_{\min }\right)
$$

Where $F$ is the crack shape parameter considering the shape and position of the crack, $\Delta \sigma$ is the stress range at the crack, and $\sigma_{\max }$ and $\sigma_{\min }$ are the maximum and minimum stresses of the cyclic load, respectively. From Eqs (13) (14):

$$
N=\int_{a_{0}}^{a_{c}} \frac{1}{C(\Delta K)^{n}} d a=\int_{a_{0}}^{a_{c}} \frac{1}{C(F \Delta \sigma \sqrt{\pi a})^{n}} d a
$$

For the direct integration of equation (15), the fatigue crack growth life can be obtained as follows:

$$
N=\left(a_{c}^{1-n / 2}-a_{0}^{1-n / 2}\right)\left[(1-n / 2) C \pi^{n / 2}(F \Delta \sigma)^{n}\right]^{-1} \text { (16) }
$$

Where $a_{0}$ is the initial crack length and $a_{c}$ is the critical 
crack length, and Eq.(16) is suitable for the material to withstand the banner load.

The fatigue life model commonly used in fracture mechanics is used for reliability analysis. That is, the fatigue life $N$ is greater than the design life $N_{C}$, and the structure is considered reliable. The limit state equation is expressed as:

$$
g(X)=N-N_{c}
$$

The initial crack length is determined by the actual measurement, and human error will be generated during the measurement process, which has a great influence on the crack propagation life and can be used as an uncertain parameter for crack propagation. As a material parameter, $C$, $n$ and $K_{I C}$ have uncertainties and dispersions, which can be used as uncertain parameters for crack propagation. The critical crack length can be obtained by the $\mathrm{K}$ criterion. The fatigue crack instability condition is:

$$
K_{\max }=F \Delta \sigma \sqrt{\pi a}=K_{I C}
$$

The upper and lower bounds of the uncertain parameters are easy to obtain. These parameters can be regarded as interval variables. The interval expression of $a_{c}$ is:

$$
a_{c}^{I}=\frac{\left(K_{I C}^{I}\right)^{2}}{\pi\left(F \Delta \sigma^{I}\right)^{2}}
$$

The interval number is used to represent the parameters in the crack propagation vector $Z=\left(C, n, K_{I C}, a_{0}, a_{c}, \Delta \sigma, N_{c}\right)$ of the titanium alloy, as shown in Tab.1.

Tab.1 Interval representation of crack growth parameters

\begin{tabular}{lcccccc}
\hline$C$ & $n$ & $K_{I C}$ & $a_{0}$ & $a_{c}$ & $\Delta \sigma$ & $N_{c}$ \\
\hline$[\underline{C}, \bar{C}]$ & {$[\underline{n}, \bar{n}]$} & {$\left[\underline{K_{I C}}, \overline{K_{I C}}\left[\underline{a_{0}}, \overline{a_{0}}\right]\right.$} & {$\left[\underline{a_{c}}, \overline{a_{c}}\right]$} & {$\left[\underline{\Delta \sigma}, \overline{\Delta \sigma}\left[\underline{N_{c}}, \overline{N_{c}}\right]\right.$}
\end{tabular}

According to the definition of reliability, the state of crack propagation is divided into a safe state and a failure state, and the boundary between the two is the limit state. The limit state equation of fatigue crack propagation from Eqs (16)(17) is expressed as

$$
Z(X)=N-N_{c}=\left(a_{c}^{1-n / 2}-a_{0}^{1-n / 2}\right)\left[(1-n / 2) C \pi^{n / 2}(F \Delta \sigma)^{n}\right]^{-1}-N_{c}
$$

\section{B. Establishment and Solution of Interval Non-probabilistic Reliability Index}

Under the influence of uncertain parameters, fatigue crack growth life $N$ has randomness and dispersion, and its variation has a certain range, which can be regarded as interval number. Fatigue crack propagation design life $\alpha$ can be considered design error to introduce dispersion coefficient $\alpha, N_{c}$ The number of intervals is expressed as $\left[N_{c}, \overline{N_{c}}\right]$.The non-probabilistic reliability index $\eta$ of crack propagation can be defined as the interval probability that the fatigue crack growth life interval $N^{I}$ is greater than or equal to the fatigue crack propagation design life interval $N_{c}^{I}$. Expressed as follows:

$$
\eta=P\left(N^{I} \geq N_{c}^{I}\right)
$$

The range of the non-probabilistic reliability index $\eta$ is $[0,1]$. When $\eta=0$, the fatigue crack growth life interval $N^{I}$ is smaller than the fatigue crack propagation design life interval $N_{c}^{I}$, and the titanium alloy material is in a failure state. When $\eta=1$, the fatigue crack growth life interval $N^{I}$ is greater than the fatigue crack propagation design life interval $N_{c}^{I}$, and the titanium alloy material is in a safe state. When $0<\eta<1$, there is no absolute size between the fatigue crack growth life interval $N^{I}$ and the fatigue crack propagation design life interval $N_{c}^{I}$. The closer $\eta$ is to 0 , the closer the titanium alloy material is to the failure state, and the closer $\eta$ is to 1 , the closer the titanium alloy material is to the safe state.

The essence of solving the non-probabilistic reliability index $\eta$ is the inequality comparison between the fatigue crack growth life $N^{I}$ and the fatigue crack propagation design life interval $N_{c}^{I}$. The algorithm refers to Chapter 1, and the interval number is calculated using the interval arithmetic method [12].

\section{INSTANCE CALCULATION}

For the example in reference[15], assume a piece of cracked plate, thickness $b=12 \mathrm{~mm}$, sheet material is titanium alloy material, yield strength $\sigma_{Y}=1009 \mathrm{MPa}$, bearing banner load $\sigma_{\text {max }}^{I}=[45,55] \mathrm{MPa}, \sigma_{\min }=0 \mathrm{MPa}$, The shape parameter $F=1$, the initial crack length $a_{0}^{I}=[0.9,1.1] \mathrm{mm}$, the fracture toughness $K_{I C}^{I}=[87.3,97.2] \mathrm{MPa} \cdot \sqrt{\mathrm{mm}}$ and the fatigue crack propagation design life $N_{c}^{I}=[20000-\alpha, 20000+\alpha]$ from the test data of the research group, combined with the actual test, the maximum range of the design life is set to 10000 30000. $\alpha$ is the dispersion coefficient of fatigue crack propagation design life, the value range is $1000 \sim 10000$, the step length is 1000 , the crack growth rate is $d a / d N=4.09 \times 10^{-10}(\Delta K)^{4.12}$, and the unit of $\Delta K$ is $\mathrm{MPa} \cdot \sqrt{\mathrm{mm}}$. Reliability analysis of fatigue crack propagation on panels.

The critical crack length $a_{c}^{I}=[0.796,1.485] \mathrm{m}$ can be obtained from the Eq.(19). The limit state equation can be obtained by the method proposed in the paper

$Z(X)=\left(a_{c}^{I^{1-4.12 / 2}}-a_{0}^{I^{1-4.12 / 2}}\right)\left[(1-4.12 / 2) \times 4.09 \times 10^{-10} \pi^{4.12 / 2}\left(\Delta \sigma^{I}\right)^{4.12}\right]^{-1}-N_{c}^{I}=0$

The reliability indexes when $\alpha$ is a different value are respectively obtained, and the calculation results are shown in Tab.2. In order to compare interval non-probabilistic reliability and probability reliability calculation results, the interval variable is transformed into a random variable using the " $3 \sigma$ " criterion and the hypothesis is subject to a normal distribution. The Monte-Carlo simulation algorithm is used to obtain a reliable probability through $10^{5}$ simulation calculations. The reliability index $\beta$ of the method is verified by the interval non-probability method. The relationship between between the Monte-Carlo method $\beta$, the calculation result $\eta$ of this paper and $\alpha$ is shown in Fig.1. It can be obtained that the calculation result of the non-probabilistic reliability method is more conservative, and as $\alpha$ increases, 
the interval of $N_{c}^{I}$ expands, so that the degree of interference with the interval of $N^{I}$ increases, and the probability reliability index decreases from $99.88 \%$ to $99.24 \%$. The decline rate is slower and faster, and the result is higher. The non-probability reliability index is reduced from $99.231 \%$ to $90.846 \%$. The titanium alloy material tends to be far away from the safe state, which is consistent with the situation in the literature[16], and conforms to the titanium alloy pressure shell. Partial safety design requirements for the structure. The feasibility of the method is verified by the interval non-probabilistic reliability analysis of fatigue crack growth of titanium alloy, which better reflects the fatigue safety performance of titanium alloy

Tab. 2 Calculation results

\begin{tabular}{cccc}
\hline$\alpha$ & $N_{c}^{I}$ & $N^{I}$ & $\eta(\%)$ \\
\hline 1000 & {$[19000,21000]$} & {$[20092,46902]$} & 99.231 \\
2000 & {$[18000,22000]$} & {$[20092,46902]$} & 98.303 \\
3000 & {$[17000,23000]$} & {$[20092,46902]$} & 97.371 \\
4000 & {$[16000,24000]$} & {$[20092,46902]$} & 96.440 \\
5000 & {$[15000,25000]$} & {$[20092,46902]$} & 95.508 \\
6000 & {$[14000,26000]$} & {$[20092,46902]$} & 94.575 \\
7000 & {$[13000,27000]$} & {$[20092,46902]$} & 93.631 \\
8000 & {$[12000,28000]$} & {$[20092,46902]$} & 92.711 \\
9000 & {$[11000,29000]$} & {$[20092,46902]$} & 91.778 \\
10000 & {$[10000,30000]$} & {$[20092,46902]$} & 90.846 \\
\hline
\end{tabular}

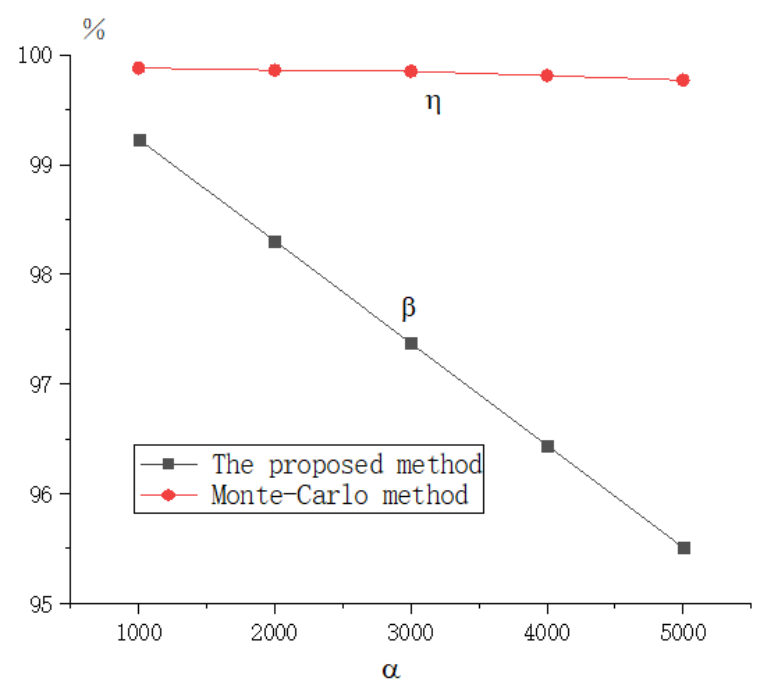

Fig.1 Comparison between the proposed method and probabilistic reliability method

\section{CONCLUSION}

For titanium alloy materials with less raw data, only the upper and lower bounds of the parameters in the crack propagation model are needed, and the interval variables can be used for uncertainty description. Based on the Paris formula and the fatigue life model, the limit state equation is established, and the fatigue life is greater than or equal to The interval probability of fatigue design life is taken as the reliability index, and the solution method is given. The example calculation shows that the non-probabilistic reliability index is more conservative than the probabilistic reliability calculation. As the dispersion coefficient $\alpha$ of the fatigue crack propagation design life interval increases, the interval interference degree increases, and the probability reliability index tends to After the steady, it showed an accelerated downward trend, while the non-probability reliability index decreased linearly, and the result became more conservative. In summary, the calculation results of the non-probabilistic reliability method are more conservative, and are safer in engineering applications, which can provide reference for fatigue analysis of titanium alloy materials. As an addition to the probabilistic reliability analysis method, the interval non-probabilistic reliability analysis method for crack propagation of titanium alloy has certain practicability in the absence of original information.

\section{REFERENCES}

[1]Chen Chenghao. Fatigue reliability analysis for titanium pressure hull of deep manned submersible[D].Shanghai Jiao Tong University, 2012. $1-2$.

[2]Sun Wencai, Yang Zichun, Huang Lian. Application of structural non-probabilistic reliability model in fracture mechanics analysis[J]. Journal of Basic Science and Engineering, 2010, 18(5): 816-822.

[3] Echard B, Gayton N, Bignonnet A. A reliability analysis method for fatigue design[J]. International Journal of Fatigue, 2014, 59(2): 292-300.

[4]Anderson T L. Fracture Mechanics: Fundamentals and Applications Surjya Kumar Maiti[J]. Mrs Bulletin, 2016, 41(8): 635-636.

[5]Hasofer A M. Convex models of uncertainty in applied mechanics : by Yakov Ben-Haim and Isaac Elishakov, studies in applied mechanics, 25 , North-Holland, Amsterdam, xviii +222 pp. Dfl. 185.00, ISBN 0-444-88406-8[J]. Structural Safety, 1992, 11(2): 147-148.

[6]Ben-Haim Y. A non-probabilistic measure of reliability of linear systems based on expansion of convex models $\star[J]$. Structural Safety, 1995, 17(2): 91-109.

[7]Elishakoff I , Cai G Q , Starnes J H . Non-linear buckling of a column with initial imperfection via stochastic and non-stochastic convex models[J]. International Journal of Non-Linear Mechanics, 1994, 29(1): 71-82.

[8]Guo Shuxiang, Zhang Ling, Li Ying. Procedures for computing the non-probabilistic reliability index of uncertain structures[J]. Chines Journal of Computationan Mechanics,2005, 22(2): 227-231.

[9]Wang Xiaojun, Qiu Zhiping, Wu Zhe. Non-probabilistic set-based model for structural reliability $[\mathrm{J}]$. Chinese Journal of Theoretical and Applied Mechanics,2007, 23(5): 641-646.

[10] Qiu Zhiping. Non probabilistic set theory convex method and its application[M]. 2005, 75-89.

[11] Jiang Chao, Zheng Jing, Han $\mathrm{Xu}$, et al. A probability and interval hybrid structural reliability analysis method considering parameters correlation[J]. Chinese Journal of Theoretical and Applied Mechanics, 2014, 46(4):591-600.

[12] Moore R E, Kearfott R B, Cloud M J. Introduction to Interval Analysis.[M]// Introduction to Interval Analysis. 2009.

[13] Cao Anxi, Cui Weicheng. Non-probabilistic reliability analysis of spherical pressure hulls based on interval number theory[J]. Shipbuilding of China, 2014(1): 38-45.

[14] Ishibuchi H, Tanaka H. Multiobjective programming in optimization of the interval objective function[J]. European Journal of Operational Research, 1990, 48(2): 219-225.

[15] Wei Zongping. Interval predition of structure fatigue crack propagation life[J]. 2013, 41(15): 61-64.

[16] Qiu Z , Wang J . Reliability study of fracture mechanics based non-probabilistic interval analysis model[J]. Fatigue \& Fracture of Engineering Materials \& Structures, 2010, 33(9): 539-548.

Benyang Tu Postgraduate of Naval Architecture and Ocean En-gineering department of Jiangsu University of Science and Technology,China.+8618260636263

Chunyang Zhao Postgraduate of Naval Architecture and Ocean En-gineering department of Jiangsu University of Science and Technology, China.+8618796087050 